\title{
Distinguishing Prostate Cancer from Hyperplasia
}

\author{
W.M. KwiateK ${ }^{a}$, A. Banaś ${ }^{a}$, K. Banaś ${ }^{a}$, M. PodgórczyK ${ }^{a}$, \\ G. Dyduch ${ }^{b}$, G. FalkenberG ${ }^{c}$, M. Gajda ${ }^{d}$ And T. Cichocki ${ }^{d}$ \\ ${ }^{a}$ Institute of Nuclear Physics, Radzikowskiego 152, 31-342 Kraków, Poland \\ ${ }^{b}$ Dept. of Pathomorphology, Collegium Medicum, Jagiellonian University \\ Grzegórzecka 16, 31-034 Kraków, Poland \\ ${ }^{c}$ HASYLAB, DESY, Notkestraße 85, 22603 Hamburg, Germany \\ ${ }^{d}$ Dept. of Histology, Collegium Medicum, Jagiellonian University \\ Kopernika 7, 31-034 Kraków, Poland
}

The prostate gland is the most common site of neoplastic disorders in men. The knowledge of the pathogenesis of benign prostate hyperplasia and prostate cancer is still under investigation. X-ray fluorescence analysis using synchrotron radiation seems to be an appropriate technique to study the elemental composition of biological tissues. The measurements presented in this paper were carried out on the L-beam line at the HASYLAB, DESY (Germany). The observed concentrations of $\mathrm{Fe}, \mathrm{Cu}, \mathrm{Mn}$, and $\mathrm{Zn}$ are significantly higher in cancerous tissues, as compared to normal and hyperplastic ones. Our results reveal also the existence of the two types of hyperplasia.

PACS numbers: 87.64.- $\mathrm{t}$

\section{Introduction}

Enlargement of the prostate is an age-related, physiological process that is unique in human tissue. The prostate gland is known as the most common site of occurrence neoplastic disorders in men. In the face of the growing impact of the various prostate diseases in terms of morbidity and mortality, the pathogenesis of benign prostate hyperplasia (BPH) and of prostate cancer remains poorly understood. This reflects the complex composition of the gland with different anatomic, cellular, and functional compartments that are differentially involved in benign and malignant disease processes.

One can easily confuse benign prostate hyperplasia with prostate cancer believing it is the initial stage of prostate cancer. However, this is not true and these above-mentioned cases are completely different. Both diseases are hard to 
distinguish from each other since they share some of the symptoms and sometimes can coexist at the same time.

Trace elements concentrations in prostate tissues are linked to the functional role of this organ and play an important role in a number of biological processes. They may also vary under different physical and chemical conditions or various physiological and pathological status, such as irradiation, lack of oxygen, injury of free radical, cancer disease, etc. For these reasons the knowledge of elemental concentrations and compositions in healthy and neoplastic tissues might help in diagnostic tests and in better understanding of the etiology and development of any abnormalities such as cancer.

In this paper we focused on determination of elemental concentrations of $\mathrm{Fe}, \mathrm{Cu}, \mathrm{Mn}$, and $\mathrm{Zn}$ in order to obtain a reliable method to distinguish hyperplasia from cancer in prostate tissues. $\mathrm{Cu}, \mathrm{Mn}$, and $\mathrm{Zn}$ are the prosthetic groups of several metalloenzymes including superoxide dismutase (SOD) which is important antioxidant enzyme in the cellular protection from reactive oxygen species (ROS) [1]. On the other hand $\mathrm{Fe}, \mathrm{Cu}$, and $\mathrm{Mn}$ may take part in Fenton reaction, in which ROS are produced that has influence to carcinogenesis in laboratory animals and in humans.

\section{Materials and methods}

The measurements were carried out on the L-beam line at the HASYLAB, DESY. The L-beam line uses the continuous X-ray spectrum produced by the bending magnet (critical energy $E_{\mathrm{c}}=16.04 \mathrm{keV}[2]$ ). A synchrotron based system was used for the detection of the X-ray fluorescence emitted from elements existing in prostate tissues specimens. Calibration models resulting from the irradiation of multielemental gelatin standard were used for the quantity investigation of trace elements. Paramount benefit of using in the present experiments monochromatic beam (energy was set at $17 \mathrm{keV}$ ) was that any scattered radiation could be altered out. This provided a much lower background than was achievable using white light, which in the past had been generally used to increase signal intensity. During the experiments polycapillaries of various shapes were employed to form the micro-beam with sizes in diameter down to $15 \mu \mathrm{m}$ in order to irradiate small area of analysed samples. The spectra were obtained by means of the multi-channel analyzer. A typical acquisition time was $300 \mathrm{~s}$ for each spectrum.

Samples were taken from patients (56- to 72-years-old) who have been operated due to prostate cancer diseases and from patients being oncologically observed on the occasion of hyperplastic process in the prostate gland. In addition the results of the analysis of trace elements content in prostate tissues of practically healthy men were used as a control group.

Analysis was performed in a controlled environment to eliminate or at least minimize contamination effects. All samples were prepared in the same way, which has been described by Kwiatek et al. [3-5]. The original hematoxylin and eosin 
(HE)-stained slides were examined by histologist to identify the pattern of samples in order to define morphological stage of tissues and localize the most interesting areas for SRIXE analysis.

\section{Results and discussions}

A combination method consisting of synchrotron radiation X-ray fluorescence spectroscopy and histochemical staining was employed to examine the detailed concentrations level of $\mathrm{Fe}, \mathrm{Cu}, \mathrm{Mn}$, and $\mathrm{Zn}$ and morphological changes in the prostate tissues. Figure 1 presents histological view of (a) hyperplasia and (b) cancerous prostate tissue. As one can see in Fig. 1a the pattern of hyperplasia is very often inhomogeneous. This inhomogeneity may be also linked with different distributions of elements mentioned above.

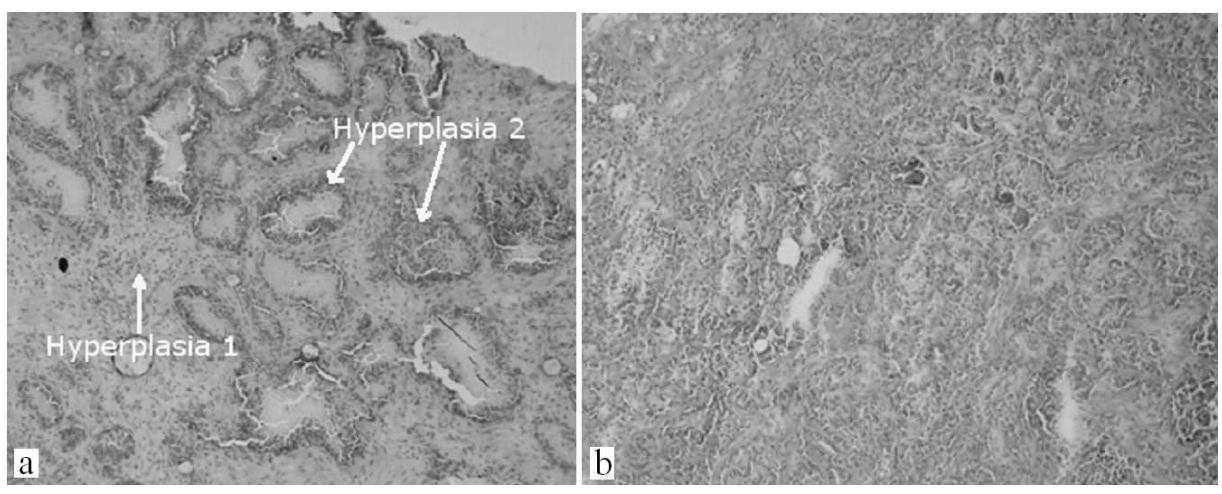

Fig. 1. Histological view of (a) hyperplasia, (b) cancerous prostate tissue.

Our analysis leads to division of hyperplasia tissues into two groups: hyperplasia-1 and hyperplasia-2. Hyperplasia-1 is the earliest stage of neoplastic changes. These tissues are mainly composed of fibrous tissues admixed with some smooth muscle. Hyperplasia-2 contained the nodules of cystically dilated glands (Fig. 1a). To check the significance of differences between the tissues in different histological states the mean values of concentrations of the individual series were investigated by the $t$-Student test. Student's distribution arises when (as in nearly all practical statistical work) the population standard deviation is unknown and has to be estimated from the data. The $t$-test for correlated samples is especially useful in research involving human or animal subjects precisely because it is so very effective in removing the extraneous effects of pre-existing individual differences.

The statistical analysis of the elemental concentrations of the different groups of prostate tissues reveals that all four elements are found at elevated levels in the cancerous tissues (see Fig. 2). The changes in mean concentrations of these 

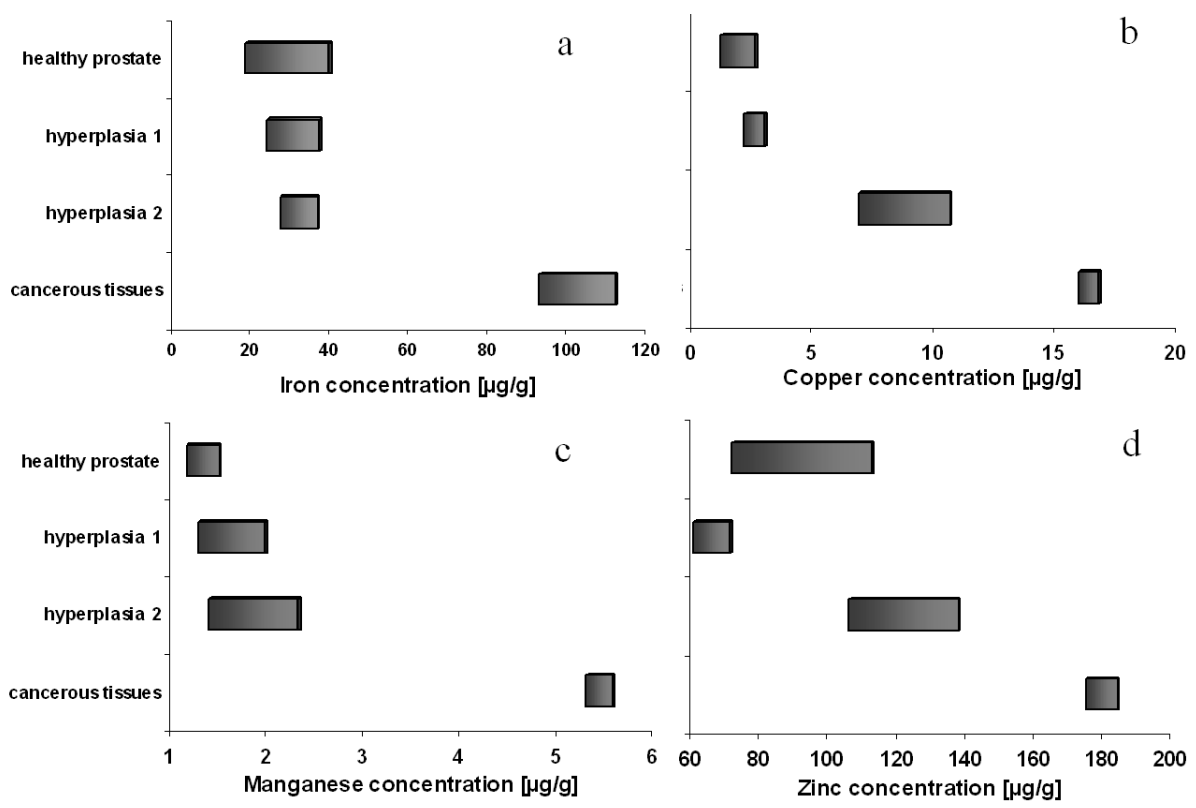

Fig. 2. Mean concentration levels for (a) Fe, (b) $\mathrm{Cu}$, (c) Mn, (d) Zn. Widths of the rectangles are equal to $2 \Delta x(\Delta x$ is an error calculated for experimental data using $t$-Student distribution).

elements in cancerous tissue are statistically proved to be significant. This fact may suggest the progression of the proliferation process in prostate cancer.

Our study indicates also that the development of hyperplasia in prostate tissues is accompanied by major changes in the level of $\mathrm{Cu}$ and $\mathrm{Zn}$ in comparison to healthy tissues. For hyperplasia-2 we observed an increase in amount of all elements, this increase is less pronounced for Fe and $\mathrm{Mn}$ and more for $\mathrm{Cu}$ and $\mathrm{Zn}$. A reasonable explanation for this discrepancy seems to be attributable to disordered cellular protection, in particular a higher level of $\mathrm{Cu}$ in hyperplasia-2 may manifest its participation in angiogenesis (new blood vessel growth).

\section{Conclusions}

Synchrotron radiation induced X-ray emission (SRIXE) in combination with histological examination is the method well suited to the direct instrumental microanalysis of prostate tissues. The data were obtained with a minimal amount of sample preparation and clearly illustrate the potential of the new highbrilliance synchrotrons for considerable improvements in the spatial resolution, data-acquisition time, detection sensitivity, and minimization of sample damage associated with these techniques.

The results of our research highlight the fact that intracellular metabolism is different in healthy and malignant tissues. The concentrations of elements such 
as $\mathrm{Fe}, \mathrm{Cu}, \mathrm{Mn}$, and $\mathrm{Zn}$ indicate whether physiological conditions are maintained. The search for prognostic markers to estimate a prostate diseases is essential for the selection of an optimal therapeutic treatment for individual patients. As some disorders types show similar histological characteristics, it is important to reveal aspects that give the pathologist hints about the protein status or even genetic information that underlies the ailment's clinical behaviour.

Our results confirmed that the elements such as $\mathrm{Fe}, \mathrm{Cu}, \mathrm{Mn}$, and $\mathrm{Zn}$ are valuable indicators and they should be considered as tracers for the identification of pathological disorders in tissues due to their strong correlation to histopathological changes.

\section{Acknowledgment}

The authors wish to thank prof. Jerzy Stachura from Collegium Medicum at the Jagiellonian University for his assistance in tissue samples classification. The measurements have been supported by HASYLAB, DESY, Hamburg, Germany under project II-02-010. This work was supported by the IHP-Contract HPRI-CT-1999-00040/2001-00140 of the European Commission.

\section{References}

[1] O.A. Ozen, M. Yaman, M. Sarsilmaz, J. Trace Elem. Med. Biol. 16, 119 (2003).

[2] http://www-hasylab.desy.de.

[3] W.M. Kwiatek, Postdoctoral Thesis (Habilitation), Institute of Nuclear Physics, PAS, Kraków 2003, Raport No. 1928/L (in Polish).

[4] W.M. Kwiatek, A. Banaś, M. Gajda, M. Gałka, T. Cichocki, Annual Report 2002, 2003, 2004, Publ.:HASYLAB, Hamburg (Germany) 2002, 2003, 2004.

[5] A. Banaś, PhD thesis, Institute of Physics, Jagiellonian University, Kraków 2005 (in Polish). 